\title{
Associations between daily steps and cognitive function among inpatients with schizophrenia
}

\author{
Li-Jung Chen ${ }^{1}$, Brendon Stubbs ${ }^{2,3}$, I-Chia Chien ${ }^{4}$, Tsuo-Hung Lan ${ }^{5,6,7,8}$, Ming-Shun Chung ${ }^{9}$, Hui-Ling Lee ${ }^{5}$, \\ Wan-Chi Hsu ${ }^{5}$ and Po-Wen $\mathrm{Ku}^{10^{*}}$
}

\begin{abstract}
Background: Walking is the fundamental component of taking steps and is the main form of physical activity among individuals with schizophrenia; it also offers a range of health benefits. This study aimed to examine the associations between daily steps and cognitive function and further explored how many steps were related to better cognitive function among inpatients with schizophrenia.
\end{abstract}

Methods: Inpatients with schizophrenia were recruited from long-stay psychiatric wards across two hospitals ( $\mathrm{n}=199$ at site 1 and $n=195$ at site 2). Daily steps were collected with an accelerometer for 7 days. Four cognitive domains (attention, processing speed, reaction time, and motor speed) were tested at site 1, and two cognitive domains (attention and processing speed) were tested at site 2. The associations of daily steps and levels of steps/day with cognitive function were tested using multivariable linear regressions separated by site. Covariates included demographic variables, weight status, metabolic parameters, and clinical state.

Results: Participants took an average of 7445 ( \pm 3442$)$ steps/day. More steps were related to better attention, processing speed, reaction time, and motor speed after multivariable adjustments. Compared with participants taking $<5000$ steps/day, those taking $\geq 5000$ steps/day showed significantly better processing speed. Participants taking $\geq 7500$ steps/day were associated with better attention, better reaction time, and better motor speed than those taking $<5000$ steps/day.

Conclusion: Daily steps are associated with better cognitive function among inpatients with schizophrenia. The optimal benefit for cognitive function among this clinical population is achieving 7500 steps/day or more.

Keywords: Physical activity, Walking, Pedometer, Psychiatric disorder, Cognition

\section{Background}

Individuals with schizophrenia tend to have higher risks of medical comorbidity and cardiovascular diseases, as well as more sedentary time and lower levels of physical activity, compared with the general population [1-3]. A

\footnotetext{
*Correspondence: powen.ku@nchu.edu.tw

${ }^{10}$ Graduate Institute of Sports and Health Management, National Chung Hsing University, 145 Xingda Rd., South Dist, Taichung 402, Taiwan Full list of author information is available at the end of the article
}

sedentary lifestyle has been linked to several health problems, such as higher body mass index, lower cardiorespiratory fitness, and worse health-related quality of life, among individuals with schizophrenia [3, 4]. In contrast, a physically active lifestyle has been reported to be beneficial for this population, including better mental health and improved cognitive function and physical health [5-8]. Consequently, increasing physical activity is recognized as a key component of the wellbeing and health of people with schizophrenia [8]. 
To date, most physical activity research and guidelines have expressed physical activity in terms of frequency, duration, and intensity parameters. Objective devices, such as pedometers, accelerometers, and wearable technology watches, provide a new opportunity to assess and communicate physical activity in the form of daily steps $[9,10]$. Most of these devices (e.g., pedometers and wearable technology watches) are simple and easy to access with relatively low cost and can easily be used in public health and clinical applications [9]. Walking, the main contributor to steps, can be performed at light, moderate, or vigorous intensity [11]. We have previously examined device-measured light and moderate-vigorous physical activity among 199 inpatients with schizophrenia [12]. The results showed positive associations between both light and moderate-vigorous physical activity and cognitive function, with a stronger relationship for light physical activity than for moderate-vigorous physical activity. Moreover, recent research has indicated that the number of steps/day is a better indicator than moderate-high intensity physical activity for cardiorespiratory fitness [13] and cognitive function [14] among patients with schizophrenia. These results suggest that recommendations to achieve a daily number of steps might be a good strategy for promoting health among patients with schizophrenia and encouraging self-management and monitoring of their activity.

Over the last two decades, step-based recommendations have emerged $[9,10,15-19]$. The common guideline of daily steps among healthy adults suggested by governments or professional organizations is achieving 10000 steps/day as the ideal goal [20-22]. Recent research has suggested that taking as few as $4400-7500$ steps/day may also contribute to health benefits $[18,19,23]$. For example, taking 4400 steps/day was associated with a reduction in mortality rate, and taking 7500 steps/day had the optimal benefit for mortality reduction among older women [19]. However, these findings tend to focus on the general population. The evidence available to answer the question 'how many steps are associated with better cognitive function among individuals with schizophrenia' is still limited.

Therefore, we reanalyzed the previous data with regard to daily steps instead of light and moderate-vigorous physical activity [12]. We also recruited more inpatients with schizophrenia from an additional hospital, used additional tests for cognitive functions to examine the associations between daily steps and various domains of cognitive function and further explored how many steps were related to better cognitive function among inpatients with schizophrenia.

\section{Methods}

\section{Participants}

Participants were recruited from long-stay psychiatric wards at two mental hospitals in Taiwan (site 1: Jianan Psychiatric Center; site 2: Tsaotun Psychiatric Center). The inclusion criteria included (1) age between 20 and 64 years; (2) diagnosis of schizophrenia by psychiatrists relying on the criteria of the Diagnostic and Statistical Manual Disorders (DSM-IV at site 1, DSM-5 at site 2); and (3) stable and on the same antipsychotic medicine for at least three months prior to inclusion in the study. We excluded participants who were not able to communicate, walk independently, or had brain injury or neurological disorders. These conditions were collected from the hospital records and diagnosed by psychiatrists. Research assistants accompanied by psychiatric nurse practitioners (site 1) and trained occupational therapists (site 2) introduced the aim and procedure of the study at each longstay psychiatric ward.

The sample size was calculated using G*Power by assuming an alpha level of 0.05 and a beta (power) of 0.8 , with a medium effect size of .15 [24]. The results showed that a sample size of 123 would be needed to achieve a significant effect. At site 1, 200 inpatients were recruited, but one of them did not sign the informed consent form. At site 2, 218 eligible inpatients were recruited. Among them, 23 inpatients were further excluded because of refusing to take part in the study or refusing to wear the accelerometer. These resulted in 199 participants at site 1 and 195 participants at site 2 with written informed consent. Data collection was completed in 2015 at site 1 and in 2019 at site 2 .

The research was approved by the Jianan Mental Hospital Institutional Review Board (site 1) and Tsaotun Psychiatric Center Institutional Review Board (site 2).

\section{Measures}

\section{Daily steps}

The hospital provided 20-min (site 1) or 30-min (site 2) stretching and walking sessions in the morning and afternoon. Inpatients were also encouraged to attend a 60-min group exercise at a frequency of 5-6 times/week at site 2 . These physical-activity promotion strategies, included in the usual care, were inpatients' daily routines.

The step data were collected at both sites using a triaxial accelerometer (wActiSleep) (ActiGraph, Pensacola, FL, USA), which has been validated and utilized in prior research $[2,25]$. Participants wore the accelerometer on the wrist of the nondominant hand all day for one week. They were asked to remove the accelerometer when taking a bath or performing water activities. The data were analyzed using ActiLife software version 6 (ActiGraph LLC, Pensacola, FL, USA). 


\section{Cognitive function}

Attention (site 1). The Cognitrone test is a subset of the Vienna Test System (VTS) and has been adopted in a prior study of people with schizophrenia [26]. It measures attention and concentration by comparing four different figures about their congruence on a computer screen [27]. The total working time was 7 minutes, and the number of correct responses was calculated, with higher values representing better attention.

Reaction time and motor speed (site 1). The reaction test is also a subset of the VTS, measuring the time reacting to visual stimuli. It requires participants to put the index finger of the dominant hand on the rest key of a working panel. Then, they have to press the reaction key as quickly as possible when specific stimuli are presented. After pressing the reaction key, they should return the finger immediately to the rest key. The mean reaction time and motor speed are recorded (in milliseconds), representing the time that elapses between the start of a visual signal and the moment the finger leaves the rest key. The rest key and the reaction key were used to distinguish between reaction and motor time [26]. The higher values indicate slower reaction time.

Processing speed (site 1). The Grooved Pegboard test measures manual dexterity and processing speed and has been employed in a prior study of patients with schizophrenia [26]. The pegboard has 25 grooved holes, and the slots are randomly positioned. It requires participants to insert pegs into the board in a fixed order from side to side and from top to bottom as quickly as possible. The amount of time from when individuals start the test until the last peg is inserted in place is recorded in seconds. Each participant was tested two times with only the right hand and the left hand. Then, the average time for the two tests was computed, with higher scores suggesting slower processing speed.

Attention (site 2). Chu's attention test assesses attention, which has shown excellent test-retest reliability (intraclass correlation coefficient $(\mathrm{ICC})=0.95)$ [28] and has been adopted in a prior study of people with schizophrenia [29]. The formal test includes 200 items and an extra 8 items for practice. There are a series of scrambled codes with the ${ }^{(*)}$ sign among the codes. Participants were asked to count the occurrences of the "*' sign. The correct responses in 10 minutes were calculated, and a higher score represents better attention.

Processing speed (site 2). Chu's hand dexterity test assesses hand dexterity and processing speed and has been utilized in a prior study in people with schizophrenia [29]. The test-retest reliability ( $\mathrm{ICC}=0.76-0.87$ ) is acceptable [30]. The test requires participants to put an iron bar into a board in a fixed hole and to insert a nut into the iron bar. Participants needed to perform the test in a fixed order as quickly as possible within 2 minutes. Each participant was tested three times with the dominant hand, the nondominant hand, and both hands. The final score was obtained by summing the numbers of iron bars and nuts for the tests, with higher scores representing better processing speed.

\section{Covariates}

Demographic variables were collected, including age, sex, years of education, smoking habits, and alcohol consumption. Behaviors of smoking and alcohol consumption were classified into two levels: 'Yes' and 'Never'. Body mass index (BMI) was computed from measured weight and height, and overweight/obesity was defined as BMI $\geq 24$ [31].

Data about clinical status profiles, including illness onset, duration of hospitalization, and the use of antipsychotic medication, were retrieved from the hospital medical records. A daily equivalent dosage of chlorpromazine was computed based on records of antipsychotic medication [32].

We collected waist circumference, systolic/diastolic blood pressure (SBP/DBP), fasting glucose (FG), serum triglycerides (TG), and high-density lipoprotein cholesterol (HDL-C) from regular health checks in the hospital. Participants were classified as 'abnormal' if they met the following conditions: (1) men with waist circumference $\geq 90 \mathrm{~cm}$ and women with waist circumference $\geq 80$ $\mathrm{cm}$; (2) SBP/DBP $\geq 130 / 85 \mathrm{mmHg}$; (3) FG $\geq 100 \mathrm{mg} / \mathrm{dl}$; (4) $\mathrm{TG} \geq 150 \mathrm{mg} / \mathrm{dl}$; or (5) men with HDL-C $<40 \mathrm{mg} / \mathrm{dl}$ and women with HDL-C $<50 \mathrm{mg} / \mathrm{dl}$ [33]. The index of metabolic syndromes was created by summing the number of abnormal metabolic parameters.

The tests were administered by psychiatric nurse practitioners (site 1) and trained occupational therapists (site 2) who were responsible for caring for the inpatients and had completed professional psychiatric training and research ethics training.

\section{Statistical analyses}

Pearson correlations were used to examine the univariate associations between daily step counts and different domains of cognitive function at each site. Multivariable linear regression analyses were performed to test the independent associations between daily step counts and cognitive function. To explore how many steps were associated with better cognitive function, the number of daily steps was categorized into four levels $(\geq 10000$, 7500-9999, 5000-7499, and <5000 steps) [10, 15, 23]. Then, the variable of step levels was organized into dummy variables, where the level of steps $<5000$ served as the reference group. All models were adjusted for age, sex, education, smoking, alcohol consumption, weight 
status, illness onset, duration of hospitalization, antipsychotic medication, and number of metabolic symptoms. The level of significance was set at .05 for all statistical analyses, which were performed with SPSS statistics 22 (IBM, New York, USA).

\section{Results}

The participants were on average aged 48 years and had been hospitalized for an average of 74.9 months with a mean illness onset of 23.9 years. The daily steps were 7,445 among inpatients with schizophrenia, with $21.6 \%$ reaching 10000 steps/day and $24.9 \%$ walking less than 5000 steps/day. Detailed information about the characteristics of the participants for each site is presented in
Table 1. Differences between the two sites were noted, indicating that participants at site 2 were older and less overweight or obese and had a lower prevalence of alcohol consumption, fewer years of schooling, fewer metabolic symptoms, more hospitalization time, and more daily steps.

Table 2 shows the univariate associations of daily steps with cognitive function among inpatients with schizophrenia separated by site. Daily steps were significantly associated with all cognitive measures at both sites, suggesting that participants with more steps/day were associated with better attention, processing speed, reaction time, and motor speed.

The results of multivariable linear regression analyses predicting cognitive function by counts and levels

Table 1 Characteristics of participants

\begin{tabular}{|c|c|c|c|}
\hline Variables/Mean \pm SD or $n(\%)$ & Site 1 & Site 2 & $p$ \\
\hline Age (year) & $44.0 \pm 9.9$ & $52.1 \pm 8.5$ & $<.001$ \\
\hline $\operatorname{Sex}(\%)$ & & & .879 \\
\hline Male & $122(61.3)$ & $121(62.1)$ & \\
\hline Female & $77(38.7)$ & $74(37.9)$ & \\
\hline Schooling (year) & $11.4 \pm 2.2$ & $10.7 \pm 2.8$ & .007 \\
\hline Smoking (\%) & & & .136 \\
\hline Yes & $85(42.7)$ & $69(35.4)$ & \\
\hline Never & $114(57.3)$ & $126(64.5)$ & \\
\hline Alcohol (\%) & & & .017 \\
\hline Yes & $33(16.6)$ & $17(8.7)$ & \\
\hline Never & $166(83.4)$ & $178(91.3)$ & \\
\hline BMI status (\%) & & & $<.001$ \\
\hline Overweight/obese & $81(40.7)$ & $43(22.1)$ & \\
\hline No & $118(59.3)$ & $152(77.9)$ & \\
\hline Chlorpromazine equivalent doses $(\mathrm{mg} / \mathrm{d})$ & $847.6 \pm 783.8$ & $836.1 \pm 713.8$ & .878 \\
\hline Duration of hospitalization (month) & $14.2 \pm 17.0$ & $137.0 \pm 91.0$ & $<.001$ \\
\hline IIIness onset (year) & $23.8 \pm 6.5$ & $23.9 \pm 8.1$ & .075 \\
\hline Number of Metabolic Symptoms & $1.7 \pm 1.3$ & $0.9 \pm 1.0$ & $<.001$ \\
\hline Daily steps (count) & $6628.1 \pm 3362.2$ & $8278.6 \pm 3329.4$ & $<.001$ \\
\hline Daily steps group (\%) & & & $<.001$ \\
\hline$\geq 10000$ & $31(15.6)$ & $54(27.7)$ & \\
\hline 7500-9999 & $51(25.6)$ & $55(28.2)$ & \\
\hline $5000-7499$ & $50(25.1)$ & $55(28.2)$ & \\
\hline$<5000$ & $67(33.7)$ & $31(15.9)$ & \\
\hline \multicolumn{4}{|l|}{ Cognitive performance } \\
\hline Site 1: Attention ${ }^{\mathrm{a}}$ (count) & $171.9 \pm 100.5$ & & \\
\hline Site 1: Processing speed ${ }^{b}$ (score) & $137.7 \pm 45.6$ & & \\
\hline Site 1: Reaction time ${ }^{c}(\mathrm{msec})$ & $661.8 \pm 480.3$ & & \\
\hline Site 1: Motor speed ${ }^{\mathrm{C}}$ (msec) & $384.8 \pm 213.4$ & & \\
\hline Site 2: Attention ${ }^{d}$ (score) & & $47.8 \pm 29.9$ & \\
\hline Site 2: Processing speed ${ }^{\mathrm{e}}$ (score) & & $129.3 \pm 33.1$ & \\
\hline
\end{tabular}

BMI body mass index

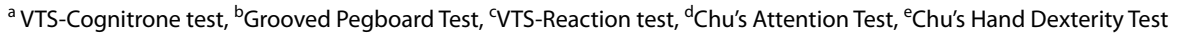


Table 2 Univariate correlations between daily steps and cognitive performance

\begin{tabular}{|c|c|c|c|c|c|c|c|c|}
\hline \multirow[t]{2}{*}{ Variables } & \multicolumn{5}{|l|}{ Site 1} & \multicolumn{3}{|l|}{ Site 2} \\
\hline & Step counts & Attention $^{a}$ & $\begin{array}{l}\text { Processing } \\
\text { speed }^{b}\end{array}$ & Reaction time $^{c}$ & Motor speed $^{c}$ & Step counts & Attention $^{d}$ & $\begin{array}{l}\text { Processing } \\
\text { speed }^{\mathrm{e}}\end{array}$ \\
\hline \multicolumn{9}{|l|}{ Site 1} \\
\hline Step counts & 1 & & & & & & & \\
\hline Attention ${ }^{a}$ & $.257^{* * *}$ & 1 & & & & & & \\
\hline Processing speed ${ }^{b}$ & $-.313^{* * *}$ & $-.412^{* * *}$ & 1 & & & & & \\
\hline Reaction time $^{c}$ & $-.245^{* * *}$ & $-.421^{* * *}$ & $.409^{* * *}$ & 1 & & & & \\
\hline Motor speed ${ }^{c}$ & $-.232^{* *}$ & $-.420^{* * *}$ & $.236^{* *}$ & $.510^{* * *}$ & 1 & & & \\
\hline \multicolumn{9}{|l|}{ Site 2} \\
\hline Step counts & & & & & & 1 & & \\
\hline Attention $^{d}$ & & & & & & $.229^{* *}$ & 1 & \\
\hline Processing speed ${ }^{e}$ & & & & & & $.301^{* * *}$ & $.417^{* * *}$ & 1 \\
\hline
\end{tabular}

of steps/day are presented in Table 3 (Supplementary Table 1 for the results of the full model). Model 1 shows that counts of steps/day remained significantly associated with all cognitive measures even after multivariable adjustments for both sites (Site $1: \mathrm{R}^{2}=.201$, Beta $=.246$, $p=.001$ for attention, $\mathrm{R}^{2}=.226$, Beta $=-.313, p<.001$ for processing speed, $\mathrm{R}^{2}=.120$, Beta $=-.267, p=.001$ for reaction time, $\mathrm{R}^{2}=.152$, Beta $=-.276, p<.001$ for motor speed; Site $2: \mathrm{R}^{2}=.271$, Beta $=.259, p=.001$ for attention, and $\mathrm{R}^{2}=.176$, Beta $=.232, p=.003$ for processing speed).
To examine how many steps were associated with better cognitive function among participants, levels of steps/ day with dummy variables were also tested (Model 2). The results showed that taking $\geq 7500$ steps/day was associated with better attention (Site 1: Beta=.230, $p=.005$ for 7500-9999 steps/day, Beta $=.266, p=.001$ for $\geq 10000$ steps/day; Site 2: Beta $=.212, p=.041$ for $7500-9999$ steps/ day, and Beta $=.280, p=.006$ for $\geq 10000$ steps/day) and better reaction time (Site 1: Beta $=-.208, p=.017$ for 7500 9999 steps/day, Beta $=-.212, p=.015$ for $\geq 10000$ steps/ day for reaction time; Beta $=-.220, p=.010$ for $7500-9999$

Table 3 Results of linear regression analyses for predicting cognitive performance by counts and levels of steps/day

\begin{tabular}{|c|c|c|c|c|c|c|c|c|c|c|c|c|}
\hline \multirow[t]{3}{*}{ Variables } & \multicolumn{8}{|l|}{ Site 1} & \multicolumn{4}{|l|}{ Site 2} \\
\hline & \multicolumn{2}{|c|}{ Attention $^{a}$} & \multicolumn{2}{|c|}{ Processing speed ${ }^{b}$} & \multicolumn{2}{|c|}{ Reaction time ${ }^{c}$} & \multicolumn{2}{|c|}{ Motor speed $^{c}$} & \multicolumn{2}{|c|}{ Attention $^{d}$} & \multicolumn{2}{|c|}{$\begin{array}{l}\text { Processing } \\
\text { speed }^{\text {e }}\end{array}$} \\
\hline & Beta & $p$ & Beta & $p$ & Beta & $p$ & Beta & $p$ & Beta & $p$ & Beta & $p$ \\
\hline \multicolumn{13}{|l|}{ Model1 } \\
\hline Step counts & .246 & .001 & -.313 & $<.001$ & -.267 & .001 & -.276 & $<.001$ & .259 & .001 & .232 & .003 \\
\hline$R^{2}$ & .201 & & .226 & & .120 & & .152 & & .271 & & .176 & \\
\hline \multicolumn{13}{|c|}{$\begin{array}{l}\text { Model } 2 \text { (ref: }<5000 \\
\text { steps/day) }\end{array}$} \\
\hline$\geq 10000$ & .266 & .001 & -.292 & $<.001$ & -.212 & .015 & -.247 & .004 & .280 & .006 & .330 & .002 \\
\hline 7500-9999 & .230 & .005 & -.288 & $<.001$ & -.208 & .017 & -.220 & .010 & .212 & .041 & .223 & .037 \\
\hline 5000-7499 & .123 & .146 & -.332 & $<.001$ & -.106 & .241 & -.165 & .063 & .083 & .389 & .265 & .008 \\
\hline$R^{2}$ & .214 & & .254 & & .105 & & .145 & & .258 & & .187 & \\
\hline
\end{tabular}

${ }^{a}$ VTS-Cognitrone test (higher values represent better attention), ${ }^{b}$ Grooved Pegboard Test (higher scores suggest slower processing speed), ${ }^{\mathrm{V}} \mathrm{VTS}$-Reaction test (higher values indicate slower reaction time and motor speed), ${ }^{d}$ Chu's Attention Test (higher scores represent better attention) ${ }^{\mathrm{e}}$ Chu's Hand Dexterity Test (higher scores represent faster processing speed)

Covariates: age, sex, schooling, smoking, drinking, BMI status, illness onset, hospitalization, chlorpromazine equivalents, number of metabolic symptoms 
steps/day, and Beta $=-.247, p=.004$ for $\geq 10000$ steps/day for motor speed) in comparison to participants taking $<5000$ steps/day. The association between levels of steps/ day and processing speed suggested that participants taking $\geq 5000$ steps/day had better processing speed than those taking $<5000$ steps/day at both sites (Site 1: Beta=$.332, p<.001$ for $5000-7499$ steps/day, Beta $=-.288, p<.001$ for 7500-9999 steps/day, and Beta=-.292, $p<.001$ for $\geq 10000$ steps/day; Site 2: Beta $=.265, p=.008$ for 5000 7499 steps/day, Beta $=.223, p=.037$ for $7500-9999$ steps/ day, and Beta $=.330, p=.002$ for $\geq 10000$ steps/day).

\section{Discussion}

To the best of our knowledge, this study is the first and largest study to explore how many steps are associated with better cognitive function among inpatients with schizophrenia. The results showed that daily steps were related to better cognitive function within each domain, including attention, reaction time, motor speed, and processing speed. Participants taking $\geq 5000$ steps/day had better performance in one cognitive domain, namely, processing speed, compared to those with $<5000$ steps/day. When participants achieved 7500 steps/day or more, better cognitive function across all domains (attention, reaction time, motor speed, and processing speed) was observed.

The mean number of daily steps was 7,445 steps/day among all participants in this study, which is much higher than the general population, which has been shown to average 4961 steps/day across all age groups in 111 countries $(n=717,527)$ [34], and higher than outpatients with schizophrenia with a mean age 26.3 years, at 5685 steps/ day $(n=62)$ [13]. One of the reasons for the high number of daily steps might be that all participants in this study were hospitalized inpatients and might be protected from daily schedules and obligations [15]. These inpatients attended 20- to 30-min walking sessions in the morning and afternoon every day at both sites. A 60-min group exercise at a frequency of 5-6 times/week was additionally provided at site 2 , which may also partly explain why more daily steps were taken among participants at site 2 than at site 1 .

Consistent with previous cross-sectional and longitudinal studies in older adults, daily steps were found to be associated with better cognitive function in some domains (attention, executive function, and subjective cognitive ability) [35, 36]. Among patients with schizophrenia, a 6-month follow-up study with a small sample size $(n=25)$ found that increased walking steps were associated with greater improvements in cognitive flexibility and executive function. This study with a larger sample size also found significant associations between daily steps and better cognitive function in several domains, suggesting that walking might be beneficial for cognitive function in patients with schizophrenia, although it is a relatively mild activity in terms of intensity [14].

Regarding the question 'How many steps/day are associated with better cognitive function among inpatients with schizophrenia?' Researchers have suggested setting minimal values of 7000-8000 steps/day for healthy adults and 6500-8500 steps/day for individuals living with disability and/or chronic illness [9, 37]. Cavero-Redondo et al. found a proactive effect on arterial stiffness within 7,500-9,999 steps/day among adults in their review study [23]. Among middle-aged adults, a cross-sectional study examined associations of steps walked per day with brain volumes measured by magnetic resonance imaging for a large sample size $(n=2354)$ [38]. The results showed that among individuals not meeting physical activity guidelines, achieving 7500 steps/day or more was associated with higher total brain volume. Another study found that older women taking 7500 steps/day had the optimal benefit for mortality reduction [19]. The current study showed that inpatients with schizophrenia taking $\geq 5000$ steps/day had better performance in one cognitive domain (processing speed). However, when participants achieved 7500 steps/day or more, better performance was found in all four cognitive domains (attention, processing speed, reaction time, and motor speed). This step range fits approximately into the cutoff point of daily steps for various health benefits among the general population, with 7500 steps/day being associated with better cognitive function among inpatients with schizophrenia.

Walking is the fundamental component of taking steps, and it is the main form of physical activity among individuals with schizophrenia. It is associated with a range of health benefits, such as weight reduction and improvement of cardiorespiratory fitness and cognitive function $[13,14,39]$. Wearable devices provide quick feedback on daily steps, are simple to use, and are easy to understand. Therefore, promoting physical activity in terms of daily steps might be a feasible and useful strategy for health promotion among patients with schizophrenia [39].

Although this study involved a relatively large sample size for people with schizophrenia and measured various cognitive domains, it is subject to some limitations, which should be borne in mind when interpreting the results. The primary limitation is that the data are cross-sectional; therefore, we are not able to infer a causal relationship between daily steps and cognitive function. The absence of the control group is also a limitation. Additionally, walking intensity may affect the step-cognition association beyond the number of walking steps. All participants in this study were individuals with established psychosis who were long-term psychiatric inpatients, and the findings may not fully generalize to less severe outpatients, limiting generalizability. To extend our findings, well-designed prospective 
cohort studies or randomized controlled trials are required to confirm/refute our exploratory data.

In summary, daily steps are associated with better cognitive function among inpatients with schizophrenia. Taking $\geq 5000$ steps/day shows a small but significant association with better cognitive function. The optimal benefit for cognitive function among inpatients with schizophrenia is achieving 7500 steps/day or more.

\section{Abbreviations}

BMI: Body mass index; FG: Fasting glucose; HDL-C: High-density lipoprotein cholesterol; ICC: Intraclass correlation coefficient; SBP/DBP: Systolic/diastolic blood pressure; TG: Triglycerides; VTS: Vienna Test System.

\section{Supplementary Information}

The online version contains supplementary material available at https://doi. org/10.1186/s12888-022-03736-2.

\section{Additional file 1.}

\section{Acknowledgements}

None

\section{Authors' contributions}

LJC: Conceptualization; Funding acquisition; Formal analysis; Roles/Writing original draft. BS: Conceptualization; Writing - review \& editing. ICC and THL: Conceptualization; Resources. MSC, HLL, and WCH: Resources; Investigation. PWK: Conceptualization; Supervision; Writing - review \& editing. All authors provided comments and conceived the study. The author(s) read and approved the final manuscript.

\section{Funding}

This work was in part supported by the Taiwan Ministry of Science and Technology (107-2410-H-028-003-MY3).

\section{Availability of data and materials}

The datasets used and/or analyzed during the current study are available from the corresponding author on reasonable request.

\section{Declarations}

\section{Ethics approval and consent to participate}

This study was approved by the Jianan Mental Hospital Institutional Review Board and Tsaotun Psychiatric Center Institutional Review Board. Written informed consent was obtained from all participants. All procedures performed in the study were in accordance with the ethical standards of the institutions and with the Declaration of Helsinki.

\section{Consent for publication}

Not applicable

\section{Competing interests}

The authors declare that they have no conflicts of interest.

\section{Author details}

${ }^{1}$ Department of Exercise Health Science, National Taiwan University of Sport, 271, Lixing Road, Taichung 404, Taiwan. ${ }^{2}$ Physiotherapy Department, South London and Maudsley NHS Foundation Trust, Denmark Hill, London SE5 8AZ, United Kingdom. ${ }^{3}$ Department of Psychological Medicine, Institute of Psychiatry, Psychology and Neuroscience, King's College London, De Crespigny Park, London, Box SE5 8AF, United Kingdom. ${ }^{4}$ Center for the Development of Teaching and Research, Bali Psychiatric Center, Ministry of Health and Welfare, 33, Huafushan Rd, Bali Distric, New Taipei City 249, Taiwan. ${ }^{5}$ Tsaotun Psychiatric
Center, Ministry of Health and Welfare, 161, Yu-Pin Rd, Nan-Tou County 542 Caotun Township, Taiwan. ${ }^{6}$ Department of Medicine, National Yang Ming Chiao Tung University, Taipei, Taiwan. ${ }^{7}$ Center for Neuropsychiatric Research, National Health Research Institutes, Miaoli, Taiwan. ${ }^{8}$ Institute of Clinical Medicine, National Yang Ming Chiao Tung University, Taipei, Taiwan. ${ }^{9}$ Jianan Psychiatric Center, Ministry of Health and Welfare, 80, Lane 870, Zhongshan Road, Tainan 717, Taiwan. ${ }^{10} \mathrm{Graduate}$ Institute of Sports and Health Management, National Chung Hsing University, 145 Xingda Rd., South Dist, Taichung 402, Taiwan.

Received: 9 May 2021 Accepted: 20 January 2022

Published online: 04 February 2022

\section{References}

1. Mitchell A, Lawrence D. Revascularisation and mortality rates following acute coronary syndromes in people with severe mental illness: comparative meta-analysis. Bri J Psychiatry. 2011;198(6):434-41.

2. Stubbs B, Chen L-J, Chung M-S, Ku P-W. Physical activity ameliorates the association between sedentary behavior and cardiometabolic risk among inpatients with schizophrenia: A comparison versus controls using accelerometry. Comprehensive Psychiatry. 2017;74:144-50.

3. Firth J, Siddiqi N, Koyanagi A, Siskind D, Rosenbaum S, Galletly C, et al. The Lancet Psychiatry Commission: A blueprint for protecting physical health in people with mental illness. Lancet Psychiatry. 2019;6(8):675-712.

4. Bueno-Antequera J, Oviedo-Caro MÁ, Munguía-Izquierdo D. Relationship between objectively measured sedentary behavior and health outcomes in schizophrenia patients: The PsychiActive project. Schizophrenia Res. 2018;197:87-92.

5. Stanton R, Happell B. A systematic review of the aerobic exercise program variables for people with schizophrenia. Curr Sports Med Rep. 2014;13(4):260-6.

6. Malchow B, Reich-Erkelenz D, Oertel-Knöchel V, Keller K, Hasan A, Schmitt $A$, et al. The effects of physical exercise in schizophrenia and affective disorders. Eur Arch Psychiatry Clin Neurosci. 2013;263(6):451-67.

7. Vancampfort D, Probst M, De Hert M, Soundy A, Stubbs B, Stroobants $M$, et al. Neurobiological effects of physical exercise in schizophrenia: $A$ systematic review. Disabil Rehabil. 2014;36(21):1749-54.

8. Stubbs B, Vancampfort D, Hallgren M, Firth J, Veronese N, Solmi M, et al. EPA guidance on physical activity as a treatment for severe mental illness: a meta-review of the evidence and Position Statement from the European Psychiatric Association (EPA), supported by the International Organization of Physical Therapists in Mental Health (IOPTMH). Eur Psychiatry. 2018:54:124-44.

9. Tudor-Locke C, Craig CL, Brown WJ, Clemes SA, De Cocker K, Giles-Corti B, et al. How many steps/day are enough? For adults. Int J Behav Nutr Phys Activity. 2011;8(1):1-17.

10. Tudor-Locke C, Hatano Y, Pangrazi RP, Kang M. Revisiting" how many steps are enough?". Med Sci Sports Exer. 2008;40(7):S537-S43.

11. Lee I-M, Buchner DM. The importance of walking to public health. Med Sci Sports Exer. 2008;40(7):S512-S8.

12. Chen L-J, Steptoe A, Chung MS, Ku P-W. Association between actigraphyderived physical activity and cognitive performance in patients with Schizophrenia. Psychol Med. 2016;46(11):2375-84.

13. Engh JA, Egeland J, Andreassen OA, Bang-Kittilsen G, Bigseth TT, Holmen $T L$, et al. Objectively assessed daily steps-not light intensity physical activity, moderate-to-vigorous physical activity and sedentary time-is associated with cardiorespiratory fitness in patients with schizophrenia. Front Psychiatry. 2019;10:82.

14. Kurebayashi Y, Otaki J. Association between altered physical activity and neurocognitive function among people with schizophrenia: A minimum 6-months' follow-up study. Comprehen Psychiatry. 2017;77:45-52.

15. Tudor-Locke C, Bassett DR. How many steps/day are enough? Sports Med. 2004;34(1):1-8.

16. Tudor-Locke C, Craig CL, Thyfault JP, Spence JC. A step-defined sedentary lifestyle index:< 5000 steps/day. Appl Physiol Nutr Metab. 2013;38(2):100-14.

17. Tudor-Locke C. Steps to better cardiovascular health: How many steps does it take to achieve good health and how confident are we in this number? Curr Cardiovasc Risk Rep. 2010;4(4):271-6. 
18. Chen S-T, Chen L-J, Ku P-W. Daily steps and health in adults: Should we walk 10,000 steps per day for optimal well-being? Taiwan J Public Health. 2020;39(1):17-26.

19. Lee I-M, Shiroma EJ, Kamada M, Bassett DR, Matthews CE, Buring JE. Association of step volume and intensity with all-cause mortality in older women. JAMA Int Med. 2019;179(8):1105-12.

20. World Health Organization. Pacific physical activity guidelines for adults: Framework for accelerating the communication of physical activity guidelines. Manila: WHO Regional Office for the Western Pacific; 2008.

21. U.S. Department of Health and Human Services. Physical Activity Guidelines for Americans. 2nd ed. Washington, DC: U.S. Department of Health and Human Services; 2018.

22. Queensland Government (Australia). 10,000 Steps Australia: Queensland Government; 2020 [Available from: https://www.10000steps.org.au/.

23. Cavero-Redondo I,Tudor-Locke C, Álvarez-Bueno C, Cunha PG, Aguiar EJ, Martínez-Vizcaíno V. Steps per day and arterial stiffness: Systematic review and meta-analysis. Hypertension. 2019;73(2):350-63.

24. Chuan CL, Penyelidikan J. Sample size estimation using Krejcie and Morgan and Cohen statistical power analysis: A comparison. J Penyelid IPBL. 2006; 7:78-86.

25. Melanson JE, Freedson PS. Validity of the Computer Science and Applications, Inc.(CSA) activity monitor. Med Sci Sports Exer. 1995;27(6):934-40.

26. Stubbs B, Ku P-W, Chung M-S, Chen L-J. Relationship between objectively measured sedentary behavior and cognitive performance in patients with schizophrenia vs controls. Schizophrenia Bull. 2017;43(3):566-74.

27. Schuhfried. Vienna Test System psychological assessment. Moedling. Austria: Schuhfried $\mathrm{GmbH} ; 2013$.

28. Lee P-S, Liu C-H, Lin H-Y, Chen Y-L, Lu W-S, Hsieh C-L. Test-retest reliability and minimal detectable change of chu's attention test in persons with chronic schizophrenia. Taiwan J Psychiatry. 2014;28(1):46-54.

29. Chen L-J, Hao JC, Ku P-W, Stubbs B. Prospective associations of physical fitness and cognitive performance among inpatients with Schizophrenia. Psychiatry Res. 2018;270:738-43.

30. Lu W, Liu C, Li P, Hsieh C, Lee P. The test-retest agreement and minimal detectable change for the Chu's Hand Dexterity Test in patients with schizophrenia. J Taiwan Occupat Ther Res Pract. 2009;5(2):93-101.

31. Pan WH, Lee MS, Chuang SY, Lin YC, Fu ML. Obesity pandemic, correlated factors and guidelines to define, screen and manage obesity in Taiwan. Obes Rev. 2008;9:22-31

32. Gardner DM, Murphy AL, O'Donnell H, Centorrino F, Baldessarini RJ. International consensus study of antipsychotic dosing. Am J Psychiatry. 2010;167(6):686-93.

33. Eckel RH, Grundy SM, Zimmet PZ. The metabolic syndrome. Lancet (London, England). 2005;365(9468):1415-28.

34. AlthoffT, Hicks JL, King AC, Delp SL, Leskovec J. Large-scale physical activity data reveal worldwide activity inequality. Nature. 2017;547(7663):336-9.

35. Calamia M, De Vito A, Bernstein JP, Weitzner DS, Carmichael OT, Keller JN. Pedometer-assessed steps per day as a predictor of cognitive performance in older adults. Neuropsychology. 2018;32(8):941-9.

36. Chen S-T, Stevinson C, Tian T, Chen L-J, Ku P-W. Accelerometer-measured daily steps and subjective cognitive ability in older adults: A two-year follow-up study. Experimental Gerontology. 2020;133:110874.

37. Tudor-Locke C, Craig CL, Aoyagi Y, Bell RC, Croteau KA, De Bourdeaudhuij I, et al. How many steps/day are enough? For older adults and special populations. Int J Behav Nutr Phys Activity. 2011;8(1):80.

38. Spartano NL, Davis-Plourde KL, Himali JJ, Andersson C, Pase MP, Maillard $P$, et al. Association of accelerometer-measured light-intensity physical activity with brain volume: The Framingham Heart Study. JAMA network open. 2019;2(4):e192745.

39. Soundy A, Muhamed A, Stubbs B, Probst M, Vancampfort D. The benefits of walking for individuals with schizophrenia spectrum disorders: A systematic review. Int J Ther Rehabil. 2014;21(9):410-20.

\section{Publisher's Note}

Springer Nature remains neutral with regard to jurisdictional claims in published maps and institutional affiliations.

Ready to submit your research? Choose BMC and benefit from:

- fast, convenient online submission

- thorough peer review by experienced researchers in your field

- rapid publication on acceptance

- support for research data, including large and complex data types

- gold Open Access which fosters wider collaboration and increased citations

- maximum visibility for your research: over 100M website views per year

At BMC, research is always in progress.

Learn more biomedcentral.com/submissions 